\title{
COMPORTAMIENTOS DE RIESGO Y NIVEL DE CONOCIMIENTO DE INFECCIONES DE TRANSMISIÓN SEXUAL EN ESTUDIANTES UNIVERSITARIOS
}

\section{RISK BEHAVIORS AND LEVEL OF KNOWLEDGE ABOUT SEXUALLY TRANSMITTED INFECTIONS IN UNIVERSITY STUDENTS}

\section{TÍTULO CORTO: COMPORTAMIENTOS DE RIESGO Y NIVEL DE CONOCIMIENTO DE} INFECCIONES DE TRANSMISIÓN SEXUAL

Lucy Margarita Villafañe-Ferrer ${ }^{1}$, Rocío Margarita González-Navarro²

Recibido en abril 05 de 2016

Aceptado en septiembre 01 de 2016

Publicado en línea en mayo 15 de 2017

\section{Resumen}

Las infecciones de transmisión sexual constituyen un problema epidemiológico y clínico de primer orden en todo el mundo por las secuelas que pueden ocasionar y por sus consecuencias económicas. Los adolescentes y jóvenes tienen mayor riesgo de presentarlas debido a factores como el inicio temprano de relaciones sexuales, la promiscuidad, entre otros. El objetivo de esta investigación fue determinar los comportamientos de riesgo y nivel de conocimiento de infecciones de transmisión sexual en estudiantes de Bacteriología de la ciudad de Cartagena. Estudio correlacional de corte transversal, en donde se aplicó un cuestionario para determinar comportamientos de riesgo y nivel de conocimiento a 128 estudiantes de Bacteriología. El 78,1 \% de los estudiantes encuestados sí ha tenido relaciones sexuales. El 55 \% ingiere bebidas alcohólicas antes de una relación sexual. A través del análisis estadístico se estableció una asociación entre la presencia de ITS con el consumo de drogas $(\mathrm{p}=0,042)$. Los estudiantes presentaron un nivel de conocimiento regular. No se encontró asociación estadísticamente significativa entre los comportamientos de riesgo y el nivel de conocimiento $(p>0,05)$. Los resultados encontrados en esta investigación evidencian la necesidad de realizar actividades de prevención para motivar cambios de comportamiento que permitan disminuir el riesgo de contagio de estas enfermedades.

Palabras clave: Enfermedades de Transmisión Sexual; Factores de riesgo; Adulto joven; Comportamiento; Conocimiento.

\section{Abstract}

Sexually transmitted infections are an epidemiologic and clinical problem of first order in all the world by effect that can produce and their economic consequences. Adolescents and young are in most risk to have these diseases by

1. Magister en Microbiología. Docente investigador Programa de Bacteriología, Corporación Universitaria Rafael Núñez. Colombia. Correo: lucy.villafane@curnvirtual. edu.co

2. Especialista en Administración de Programas de Desarrollo Social. Candidata a Magister en Desarrollo Social. Docente Programa de Bacteriología, Corporación Universitaria Rafael Núñez. Colombia. Correo: rocio.gonzalez@curnvirtual.edu.co 
facts such as premature sexual relationships and promiscuity. The objective of this investigation was to determine risk behaviors and level of knowledge about sexually transmitted infections in students' community. Correlational cross-sectional study. A questionnaire was applied to determine risk behaviors and level of knowledge about sexually transmitted infections to 128 students' community. In this study was found $78.1 \%$ of students had sexual relationships. $55 \%$ of students drink alcohol before a sexual relationship. By means of statistical analysis was found association between sexually transmitted infections and drug use $(\mathrm{p}=0.042)$. Students had a regular level of knowledge. It not was found association between risk behaviors and level of knowledge ( $p>0.05$ ). Results found in this investigation demonstrate the necessity of making activities for prevention of these infections and motivating changes of behaviors for reducing risk of contagion of these infections.

Keywords: sexually transmitted diseases; risk factors; young adult; behavior; knowledge.

\section{INTRODUCCIÓN}

L as infecciones de transmisión sexual (ITS) son enfermedades producidas por bacterias, hongos, virus y parásitos; en la mayoría de los casos la transmisión sexual tiene importancia epidemiológica; sin embargo, en algunas de casos, este modo de transmisión no es el más importante. Estas infecciones constituyen un problema epidemiológico y clínico de primer orden en todo el mundo por las secuelas que pueden ocasionar (infertilidad, enfermedad pélvica inflamatoria, cáncer anogenital, trastornos psicosexuales, etc.) y por las consecuencias económicas derivadas razón por la cual se debe priorizar en la prevención para disminuir la incidencia de estas infecciones ${ }^{1-3}$. Para tal fin, se pueden tomar medidas que se basen, entre otros aspectos, en la educación sanitaria, con el fin de limitar los comportamientos de riesgo, puesto a que estas infecciones no se limitan a grupos especiales sino que, al contrario, cualquier persona está expuesta y puede contraerlas ${ }^{4,5}$.

Los adolescentes y jóvenes pertenecen a los grupos poblacionales que tienen mayor riesgo de presentar ITS debido a factores como el inicio temprano de relaciones sexuales, la promiscuidad, los conocimientos superficiales sobre ITS, entre otros; lo que representa para ellos un verdadero problema de salud ${ }^{1,6}$. Por lo tanto, es importante la evaluación y comprensión de las conductas de riesgo en estas poblaciones porque permite orientar la utilización de recursos en el desarrollo de estrategias de intervención idóneas, con el fin de prevenir, controlar y reducir el riesgo de adquirir estas infecciones ${ }^{7-9}$. Para esta investigación se entiende como 'comportamientos sexuales de riesgo' las diferentes maneras como se lleva a cabo la actividad sexual y el conocimiento que se tiene al respecto.

Según el Ministerio de Protección Social ${ }^{10}$, cada hora en Colombia, a diez colombianos se les diagnostica una ITS, principalmente Virus del Papiloma Humano, sífilis, herpes y gonorrea. En cuanto a edad en Colombia, el mayor rango de afectados está entre los 15 y 30 años de edad. En jóvenes colombianos se han reportado comportamientos que incrementan el riesgo de adquirir estas infecciones, principalmente: las relaciones sexuales sin protección, múltiples parejas sexuales, el no uso de preservativo y el inicio temprano de las relaciones sexuales ${ }^{11}$.

Diversos estudios se han realizado para evaluar los comportamientos de riesgo asociados a ITS en diferentes poblaciones, tales como en estudiantes de secundaria, adolescentes, mujeres que ejercen la prostitución y en estudiantes de medicina; sin embargo, no existían investigaciones sobre este tópico en estudiantes de Bacteriología ${ }^{12-16}$. Los estudiantes universitarios de la ciudad de Cartagena, por los factores mencionados anteriormente, son un grupo con riesgo de presentar una ITS.

Las instituciones de educación superior deben velar por la formación integral de sus estudiantes en los aspectos intelectuales, sociales y profesionales, para que tengan un adecuado desempeño profesional y un desarrollo humano sostenible; dentro de este proceso se incorporan actividades ligadas al fomento y promoción de la salud. Por esta razón, es de gran relevancia evaluar en ellos el nivel de conocimiento y los comportamientos de riesgo asociados a ITS. 


\section{MATERIALES Y MÉTODOS}

Se realizó un estudio correlacional de corte transversal durante el año 2012 en 128 estudiantes de un programa de bacteriología de una institución de educación superior de la ciudad de Cartagena. Dichos estudiantes fueron seleccionados por muestreo aleatorio estratificado, teniendo en cuenta el número de estudiantes por semestre. A estos estudiantes, previo consentimiento informado, se les aplicó un cuestionario diseñado y estructurado por los investigadores, teniendo en cuenta las preguntas validadas de otros autores y la encuesta Nacional de Demografía y Salud 2010 $0^{17-19}$. El cuestionario fue respondido individualmente, de forma voluntaria y anónima.

El cuestionario estaba constituido por 21 preguntas. En la primera parte del cuestionario se recogen datos sociodemográficos; la segunda parte, preguntas de conocimiento sobre ITS; y la tercera parte está conformada por preguntas relacionadas con los comportamientos de riesgo, y en una cuarta parte se evalúan otros aspectos como padecimiento de ITS y el dato sobre si han recibido información al respecto. El instrumento fue aplicado por los responsables de la investigación.

Para la valoración del nivel de conocimiento (NC) se aplicaron 8 preguntas sobre ITS; se contabilizó teniendo en cuenta una escala según las respuestas correctas: Bueno (5-6), Regular (3-4) y Deficiente (0-2). De igual forma, para valorar los comportamientos sexuales, el grupo investigador clasificó a los estudiantes en bajo y alto riesgo teniendo en cuenta las respuestas dadas. Para analizar los resultados obtenidos se utilizó el software estadístico SPSS versión 19 para Windows.

\section{Declaración sobre aspectos éticos}

Para su ejecución, esta investigación tuvo en cuenta las normas éticas de la Declaración de Helsinki y la Resolución N 8430 de 1993 del Ministerio de Salud y Protección Social de la República de Colombia ${ }^{20,21}$. Se solicitó el consentimiento de los estudiantes para que pudieran participar en la investigación. Esta investigación fue realizada por profesionales con conocimiento y experiencia para cuidar la integridad del ser humano. De igual forma, se protegió la privacidad de los participantes.

\section{RESULTADOS}

En este estudio participaron estudiantes de primero a octavo semestre, de los cuales, la mayoría son de sexo femenino (113/128; 88,3\%; IC95\% 81,6 - 92,8) con un promedio de edad de 20,61 $\pm 2,21$ años.

El 97,7\% (125/128; IC95\%: 93,3 - 99,2) refirieron ser heterosexuales y el 2,3\% (3/128; IC95\%: 0,8 - 6,7) homosexuales.

En cuanto al estado civil: el 85,9 \% (110/128; IC95\% 78,9 - 90,9) eran solteros; el 7,8\% (10/128; IC95\% 4,3 - 13,8) casados; el 5,5\% (7/128; IC95\% 2,7- 10,9) en unión libre y el 6,3 \% (8/128; IC95\% 3,2- 11,9) divorciados.

Los resultados relacionados con los comportamientos de riesgo se encuentran consignados en la tabla 1. Entre estos, se logró identificar que el 78,1\% (100/128) de los estudiantes ha tenido relaciones sexuales. Al evaluarse las preferencias en cuanto al tipo de relación genital, el $68 \%(68 / 100)$ de los estudiantes expresó que preferían la de penetración vaginal. El uso de bebidas alcohólicas antes de una relación sexual fue mencionado por el $55 \%$ $(55 / 100)$ de los encuestados. Solo el $4 \%(4 / 100)$ de los participantes de esta investigación reportó utilizar drogas antes de una relación sexual.

Otro aspecto importante de los resultados fue la utilización de preservativos durante una relación sexual, de donde se concluyó que el 95\% (95/100) de los encuestados sí los utilizan.

El 78,1\% (100/128; IC95\%: 70,2 - 84,4) de los estudiantes que participaron en el estudio afirman haber iniciado sus actividades sexuales. El 68\% de los estudiantes que han tenido relaciones sexuales (68/100; IC95\% 58,3 - 76,3) refirieron tener una pareja en el momento de responder el cuestionario (Figura 1). De los estudiantes de Bacteriología, que han tenido relaciones sexuales, el $76 \%$ (76/100; IC95\% 66,8 - 83,3) tenía edades entre 15 y 19 años cuando tuvieron su primera relación sexual (Figura 2). 
Tabla 1. Aspectos para evaluar los comportamientos de riesgo.

\begin{tabular}{|c|c|c|c|}
\hline \multicolumn{2}{|l|}{ Aspecto investigado } & \multirow{2}{*}{$\begin{array}{c}\text { Respuestas } \\
100 / 128\end{array}$} & \multirow{2}{*}{$\begin{array}{c}\text { Porcentaje IC } \mathbf{9 5 \%} \\
78,1 \% ;(70,2-84,4)\end{array}$} \\
\hline Has tonido rolaciones coyulalos & Sí: & & \\
\hline 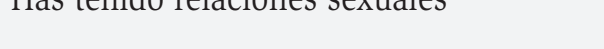 & No: & $28 / 128$ & $22,7 \% ;(16,3-30,6)$ \\
\hline \multirow[b]{2}{*}{ Fidelidad a la Pareja } & Fácil de cumplir: & $122 / 128$ & $95,3 \% ;(90,2-97,8)$ \\
\hline & $\begin{array}{l}\text { Difícil de } \\
\text { cumplir: }\end{array}$ & $6 / 128$ & $4,7 \% ;(2,2-9,9)$ \\
\hline \multirow{4}{*}{$\begin{array}{l}\text { Opinión acerca de la protección de los } \\
\text { preservativos }\end{array}$} & Protegen: & $44 / 128$ & $34,4 \% ;(26,7-43)$ \\
\hline & $\begin{array}{l}\text { No siempre } \\
\text { protegen }\end{array}$ & $75 / 128$ & $58,6 \% ;(49,9-66,8)$ \\
\hline & No protegen & $2 / 128$ & $1,6 \% ;(0,4-5,5)$ \\
\hline & No sabe & $7 / 128$ & $5,4 \% ;(2,7-10,9)$ \\
\hline \multirow{3}{*}{ Tipo de relación genital } & $\begin{array}{l}\text { Penetración } \\
\text { vaginal }\end{array}$ & $68 / 100$ & $68 \%(58,3-76,3)$ \\
\hline & $\begin{array}{c}\text { Penetración } \\
\text { vaginal y sexo } \\
\text { oral } \\
\end{array}$ & $28 / 100$ & $28 \%(20,1-37,5)$ \\
\hline & $\begin{array}{l}\text { Penetración } \\
\text { vaginal y anal, } \\
\text { sexo oral }\end{array}$ & $4 / 100$ & $4 \%(1,6-9,8)$ \\
\hline \multirow{2}{*}{$\begin{array}{l}\text { Uso de preservativos durante la relación } \\
\text { sexual }\end{array}$} & Sí & $95 / 100$ & $95 \%(88,8-97,9)$ \\
\hline & No & $5 / 100$ & $5 \%(2,2-11,2)$ \\
\hline \multirow{2}{*}{$\begin{array}{l}\text { Consumo de alcohol antes de tener una } \\
\text { relación sexual }\end{array}$} & Sí & $55 / 100$ & $55 \%(45,2-64,4)$ \\
\hline & No & $45 / 100$ & $45 \%(35,6-54,8)$ \\
\hline \multirow{2}{*}{$\begin{array}{l}\text { Consumo de drogas (marihuana, cocaína) } \\
\text { antes de tener una relación sexual }\end{array}$} & Sí & $4 / 100$ & $4 \%(1,6-9,8)$ \\
\hline & No & $96 / 100$ & $96 \%(90,2-98,4)$ \\
\hline
\end{tabular}




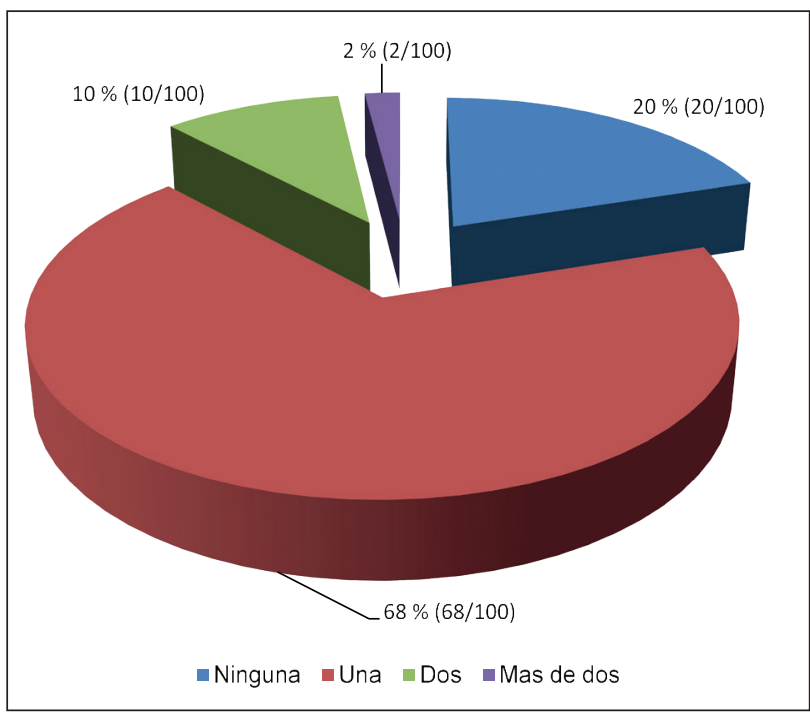

Figura 1. Distribución porcentual del número de parejas sexuales el último año

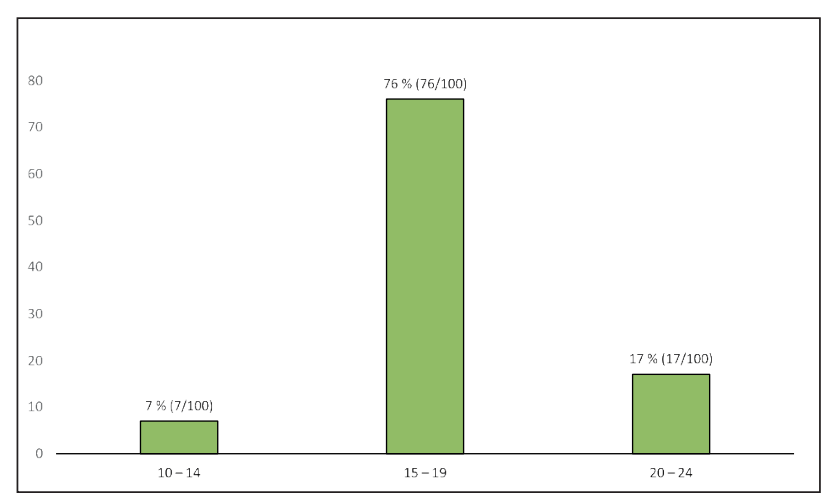

Figura 2. Distribución porcentual de la edad de inicio de las relaciones sexuales

En la población estudiada no se encontró una asociación estadística entre el inicio de las relaciones sexuales antes de los 15 años y el padecimiento de ITS $(p=0,544)$.

Tabla 2. Aspectos relevantes para evaluar el nivel de conocimiento sobre ITS/VIH/SIDA.

\begin{tabular}{|l|c|c|c|}
\hline \multicolumn{1}{|c|}{ Aspecto investigado } & & Respuestas & $\begin{array}{c}\text { Porcentaje (\%) } \\
\text { IC 95\% }\end{array}$ \\
\hline \multirow{2}{*}{ Definición de ITS } & Correctas: & 81 & $63,3 \% ;(54,7-71,1)$ \\
\cline { 2 - 4 } & Incorrectas: & 47 & $36,7 \% ;(28,9-45,3)$ \\
\hline \multirow{2}{*}{ Cómo se evita una ITS/VIH/SIDA } & Correctas: & 45 & $35,2 \% ;(27,4-43,8)$ \\
\cline { 2 - 4 } & Incorrectas: & 83 & $64,8 \% ;(56,3-72,6)$ \\
\hline \multirow{2}{*}{$\begin{array}{l}\text { Condiciones que favorecen el contagio } \\
\text { de ITS/VIH/SIDA }\end{array}$} & Correctas: & 68 & $53,1 \% ;(44,5-61,6)$ \\
\cline { 2 - 4 } Enfermedades consideradas ITS & Incorrectas: & 60 & $46,9 \% ;(38,5-55,5)$ \\
\hline \multirow{2}{*}{ Consecuencias de una ITS no tratada } & Correctas: & 45 & $35,2 \% ;(27,4-43,8)$ \\
\cline { 2 - 4 } & Incorrectas: & 83 & $64,8 \% ;(56,3-72,6)$ \\
\cline { 2 - 4 } & Correctas: & 54 & $42,2 \% ;(34-50,9)$ \\
\hline \multirow{2}{*}{ Personas en riesgo de presentar SIDA } & Incorrectas: & 74 & $57,8 \% ;(49,2-66)$ \\
\cline { 2 - 4 } & Correctas: & 108 & $84,4 \% ;(77,1-89,7)$ \\
\cline { 2 - 4 } & Incorrectas: & 20 & $15,6 \% ;(10,4-22,9)$ \\
\hline
\end{tabular}

Los aspectos para evaluar el NC aparecen en la Tabla 2, entre ellos se destaca: cómo se evita una ITS. Al respecto, se encontró que el $35,2 \%$ (45/128) de los estudiantes que respondieron de forma correcta; entre las respuestas más frecuentes están: no teniendo relaciones sexuales, teniendo una pareja estable y usando preservativo. Con relación a la pregunta sobre las condiciones que favorecen el contagio de las ITS, el 53,1\% (68/128) respondió correctamente y entre sus respuestas dadas indicaron: tener varios compañeros sexuales del sexo opuesto o de ambos sexos, no usar preservativos al tener relaciones sexuales con desconocidos, entre otras. Con respecto a las consecuencias de una ITS no tratada, el 42,2\% (54/128) de los estudiantes respondió correctamente; entre las principales respuestas figuran: infertilidad en el hombre, infertilidad en la mujer, aborto, parto prematuro y cáncer en genitales. Al evaluar qué personas están en riesgo de presentar SIDA, el 84,4\% (108/128) expresó que es una 
enfermedad que le puede dar a cualquier persona sin importar su orientación sexual.

En cuanto a la medición del $\mathrm{NC}$, de manera general se puede decir que el 41,4 \% (52/128; IC95\% 32,5 - 49,3) de los estudiantes tiene un NC regular sobre ITS (ver tabla 3 ).

Tabla 3. Nivel de Conocimiento sobre ITS.

\begin{tabular}{|l|c|c|}
\hline $\begin{array}{c}\text { Nivel de } \\
\text { conocimiento }\end{array}$ & Frecuencia & Porcentaje (\%) \\
\hline Bueno (5- 6) & 24 & 18 \\
\hline Regular (3 -4) & 52 & 41,4 \\
\hline Deficiente (0 - 2) & 52 & 40,6 \\
\hline Total & 128 & 100 \\
\hline
\end{tabular}

No se encontró asociación estadística entre el nivel de conocimiento y los comportamientos de riesgo $(\mathrm{p}=0,622)$ (ver tabla 4).

El $10 \%$ de los estudiantes que han tenido relaciones sexuales (10/100; IC95\% 5,5 - 17,4) contestó haber presentado una ITS. La más frecuente en los estudiantes de Bacteriología fue la Infección por Gardnerella vaginalis con producción de Enfermedad Inflamatoria Pélvica $(5 / 10)$; le siguen en orden de frecuencia: infecciones por Candida albicans $(2 / 10)$ y Condilomas, Hepatitis B e infección por Trichomonas vaginalis $(1 / 10$, cada uno). De los casos de ITS mencionados, 2 presentaron infecciones mixtas (Infección por G. vaginalis - Infección por C.albicans y Condiloma - Infección por G. vaginalis).

Al evaluar si los estudiantes sabían que su pareja sexual presentaba o había presentado una ITS, el 100\% de los encuestados respondió que desconocía esa información.

A través del análisis estadístico realizado se determinó una asociación entre la presencia de ITS con el consumo de drogas $(p=0,042)$. Por otro lado, no se encontró asociación entre la presencia de ITS con el uso de preservativo y consumo de alcohol ( $\mathrm{p}=0,092$ y $\mathrm{p}=0,752$, respectivamente).

Finalmente, se evaluaron los medios por los cuales los estudiantes han recibido información sobre ITS; el 45,3\% (58/128; IC95\% 36,95\% - 53,95\%) manifiesta haberla recibido por medios de comunicación como televisión o internet, mientras que el 54,7\% (70/128; IC95\% 46,05\% $63,05 \%$ ) la han recibido a través de plegables, sus padres o algún amigo; un 4,7\% (6/128; IC95\% 2,17\% - 9,85\%) no ha recibido información sobre ITS.

Tabla 4. Nivel de conocimiento versus comportamientos de riesgo de ITS/SIDA

\begin{tabular}{|l|c|c|c|}
\hline \multirow{2}{*}{ Nivel de Conocimiento } & \multicolumn{2}{|c|}{ Comportamientos de riesgo } & \multirow{2}{*}{ Total } \\
\cline { 2 - 4 } & Alto Riesgo & Bajo Riesgo & 24 \\
\hline Bueno (5-6) & 12 & 12 & 52 \\
\hline Regular (3-4) & 23 & 29 & 52 \\
\hline Deficiente (0-2) & 20 & 32 & 128 \\
\hline Total & 55 & 73 & \\
\hline
\end{tabular}

$X^{2}=0,949 p=0,622$

\section{DISCUSIÓN}

La prevención de las ITS es de extrema importancia debido a que estas enfermedades pueden provocar desde serias secuelas hasta la muerte de un individuo. Por tal razón, es primordial evaluar comportamientos de riesgo y nivel de conocimiento sobre ITS para poder definir poblaciones sobre las cuales plantear medidas de prevención ${ }^{22,23}$.
En la realización de este estudio el 97,7\% (125/128; IC95\%: 93,3 - 99,2) de los encuestados refirió ser heterosexual y el otro 2,3 \% (3/128; IC95\%: 0,8 - 6,7) homosexual. Frecuencias menores fueron reportadas por Campo et al (año) en estudiantes adolescentes de Santa Marta, en donde el $84,1 \%$ se consideró heterosexual y el 0,6\% homosexual ${ }^{24}$. Es importante mencionar que, a pesar de ser este cuestionario anónimo, las preguntas 
relacionadas con la orientación sexual pueden ser consideradas comprometedoras para algunos estudiantes; y por tal razón se tiene que considerar cierto sesgo atribuible al entrevistado.

En cuanto a la actividad sexual, el 78,1\% (100/128; IC95\% 70,2 - 84,4) de estudiantes sí ha tenido relaciones sexuales. En un estudio realizado en la Universidad Estatal de Londrina, Brasil, en estudiantes de Enfermería y Medicina, se encontró una frecuencia de $70,4 \%{ }^{16}$, resultado similar al reportado en los estudiantes de Bacteriología. Al evaluarse las preferencias en cuanto al tipo de relación genital, el 68\% (68/100; IC95\% 58,3 - 76,3) de los estudiantes expresó que preferían las de penetración vaginal, frecuencia menor a la reportada por Arias et $\mathrm{al}^{25}$ (año) en estudiantes universitarios de Cali $(91,2 \%)$. Es importante tener en cuenta que cuando no se utiliza preservativo, la penetración vaginal es una de las mayores vías de transmisión de ITS y VIH ${ }^{26}$.

El uso de bebidas alcohólicas antes de una relación sexual fue mencionado por el $55 \%$ (55/100; IC95\% $45,2-64,4)$ de los encuestados. Este resultado es similar a lo encontrado en universitarios de Alicante España (50 $\%)$; sin embargo, fue mayor al reportado en jóvenes que asisten a sitios nocturnos de Portugal $(35,2 \%)^{27,28}$. Este comportamiento es de alto incidencia, debido a los efectos de desinhibición y disminución del riesgo percibido que el alcohol produce. Lo anterior permite que los jóvenes asuman conductas (por ejemplo, no usar preservativos) que los dejan totalmente expuestos frente a una ITS como el VIH y al riesgo de embarazos no deseados ${ }^{29}$.

El 4\% (4/100; IC95\% 1,6- 9,8) de los encuestados de esta investigación reportó utilizar drogas antes de una relación sexual. El uso de drogas en estudiantes de Bacteriología fue menor al compararlo con frecuencias determinadas por otros autores ${ }^{23,27,28}$. El uso de drogas antes de una relación sexual es también considerada una conducta de riesgo al momento de tener relaciones sexuales sin protección ${ }^{29,30}$.

La utilización de preservativos durante una relación sexual se encontró en el 95\% (95/100; IC95\% 88,8 $97,9)$ de los estudiantes evaluados. Frecuencias inferiores fueron reportadas por Hernández y Cruz ${ }^{31}$, en estudiantes universitarios $(48,97 \%)$ de la Universidad Veracruzana, Veracruz- México. Otros autores ${ }^{16,28}$ también reportaron frecuencias menores. Aunque en esta investigación una alta frecuencia de estudiantes utiliza el preservativo, se puede vislumbrar que existe un pequeño grupo de estudiantes con riesgo de contraer ITS; no solo es necesario tener las competencias para utilizar el preservativo sino que también es necesario que estén dispuestos a usarlo y crean que el condón sí sirve para prevenir las ITS y, especialmente, el VIH/ SIDA $^{32}$.

De los estudiantes que participaron en el estudio, el 78,1\% (100/128; IC95\%: 70,2 - 84,4) afirma haber iniciado sus actividades sexuales. El $68 \%$ de los estudiantes que ha tenido relaciones sexuales (68/100; IC95\% 58,3 - 76,3) tenía pareja sexual cuando respondió el cuestionario. Estos resultados son similares a los reportados en estudiantes de Enfermería y Medicina en Brasil en los que la frecuencia de estudiantes que han iniciado relaciones sexuales fue $70,4 \%$ y el $71,9 \%$ tuvo una pareja sexual en el último año ${ }^{16}$. De los estudiantes de Bacteriología, que han tenido relaciones sexuales, el 76 \% (76/100; IC95\% 66,8 - 83,3) respondió tener entre 15 y 19 años cuando tuvo su primera relación sexual. Resultados análogos se presentaron en la ciudad de Cali, en donde Arias et al (año publicación), encontraron que el 76,5 \% de los estudiantes universitarios tuvo su primera relación sexual a una edad promedio de 16 años ${ }^{25}$.

Según el análisis estadístico de la población estudiada, no se estableció asociación entre el inicio de las relaciones sexuales antes de los 15 años y el padecimiento de ITS $(p=0,544)$. Aun cuando en la población estudiada estos dos aspectos no estaban asociados, es importante mencionar que la iniciación sexual a edades cada vez más tempranas conlleva a un mayor número de parejas sexuales y, por ende, un incremento en el riesgo de contraer una ITS ${ }^{22}$.

En cuanto a la medición del NC, el 41,4\% (52/128; IC95\% 32,5 - 49,3) de los estudiantes, tienen un NC regular sobre ITS; esta frecuencia es menor que la encontrada por Corona y Ortega, en adolecentes (NC: $51,9 \%$ ) del municipio de Miranda - Venezuela ${ }^{6}$. El NC encontrado en los estudiantes de Bacteriología indica que existe cierto grado de desconocimiento sobre las ITS, por lo cual se deben realizar actividades de prevención tendientes a mejorar esta situación y, por ende, disminuir el riesgo de contagio ${ }^{6}$.

El 10\% de los estudiantes que han tenido relaciones sexuales contestó afirmativamente ante la presencia de una ITS (10/100; IC95\% 5,5 - 17,4). Escalante et al (año) encontraron el mismo porcentaje de padecimiento de ITS entre adultos jóvenes de la ciudad de Lima. Aunque el porcentaje de padecimiento de ITS en estudiantes de Bacteriología es bajo, es necesario extremar medidas preventivas para crear mayor conciencia sobre este 
problema, debido a que es precisamente en estas edades donde la percepción del riesgo sobre contagio de ITS es menor $^{30}$.

Es importante mencionar que el $100 \%$ de los estudiantes respondió que desconoce si sus parejas sexuales tienen o tuvieron una ITS, situación que también es considerada riesgosa.

En los estudiantes evaluados ninguno recibió información sobre ITS por conferencias o una persona experta en el tema. Es importante que a los jóvenes se les proporcione información sobre las ITS, esta conducta parte de la hipótesis de que los individuos con un conocimiento apropiado evitarán conductas de riesgo y tomarán medidas preventivas ${ }^{33,34}$. Sin embargo, la sola información no es suficiente para motivar cambios de comportamiento; sino que se debe concientizar y sensibilizar hacia la percepción del riesgo ${ }^{30}$. Los resultados encontrados de este estudio permitieron determinar que en esta población estudiantil se deben realizar actividades tendientes a brindar información sobre ITS y motivar cambios de comportamiento que permitan disminuir el riesgo de contagio de estas enfermedades.

\section{DECLARACIÓN SOBRE CONFLICTO DE INTERESES}

Esta investigación fue efectuada con recursos propios. Los autores de este artículo declaran que no se presentaron conflictos de intereses durante su realización.

\section{REFERENCIAS BIBLIOGRÁFICAS}

1. Vives A, Sarquella G. Infecciones de transmisión sexual. Medicine. 2010; 10(61): 4202-11.

2. Satterwhite C, Torrone E, Meites E, Dunne E, Mahajan $\mathrm{R}$, Bañez Ch, et al. Sexually Transmitted Infections Among US Women and Men: Prevalence and Incidence Estimates, 2008. Sexually Transmitted Diseases. 2013; 40(3): 187-93.

3. Pérez L, Santos M, De la Paz Y. Comportamiento de las infecciones de transmisión sexual en adolescentes. Acta Médica del Centro. 2014; 8(3): 117-20.

4. Buhi E, Klinkenberger N, McFarlane M, Kachur R, Daley E, Baldwin J, et al. Evaluating the Internet as a Sexually Transmitted Disease Risk Environment for Teens: Findings From the Communication, Health, and Teens Study. Sexually Transmitted Diseases. 2013; 40(7): 528-33.

5. Díez M, Díaz A. Infecciones de Transmisión Sexual: Epidemiología y Control. Rev Esp Sanid Penit. 2011; 13: 58-66.
6. Corona J, Ortega J. Comportamiento sexual y conocimiento sobre infecciones de transmisión sexual en estudiantes venezolanos de un liceo del municipio de Miranda MEDISAN. 2013; 17(1):78-85.

7. Galindo J, Tello IC, Mueses HF, Duque JE, Shor-Posner G, Moreno G. VIH y Vulnerabilidad: una comparación de tres grupos en Cali, Colombia. Rev. Fac. Nac. Salud Pública. 2011; 29(1): 25-33.

8. Bermudez MP, Ramiro MT, Uribe- Rodriguez AF, Sierra JC, Buela Casal G. Knowledge, misconceptions, selfefficacy and attitudes regarding HIV: Cross-cultural assessment and analysis in adolescents. Int J Clin Health Psychol. 2012; 12 (2): 235-49.

9. Martínez J, Maya N, Parada L, Duarte K. Diferencias de las prácticas sexuales entre hombres y mujeres, en estudiantes universitarios durante el primer semestre de 2013. Duazary. 2013; 10(2):112-18

10. Villegas A, Tamayo L. Prevalencia de infecciones de transmisión sexual y factores de riesgo para la salud sexual de adolescentes escolarizados, Medellín, Colombia, 2013. Iatreia. 2016. 29(1):5-17.

11. Girón SL, Palacio H, Mateus JC. HIV sexual risk behaviors in youth 15-24 years of age in Cali, Colombia: Do differences exist among neighborhoods?. Colomb Med. 2013; 44(2): 72-9.

12. Sonnenberg P, Clifton S, Beddows S, Field N, Soldan $\mathrm{K}$, Tanton C, et al. Prevalence, risk factors, and uptake of interventions for sexually transmitted infections in Britain: findings from the National Surveys of Sexual Attitudes and Lifestyles (Natsal). Lancet. 2013; 382: 1795-806.

13. Mayer K, Bush T, Henry K, Overton T, Hammer J, Richardson J, et al. Ongoing sexually transmitted disease acquistion and risk taking behavior among U.S. HIV-infected patients in primary care: implications for prevention interventions. Sex Transm Dis. 2012; 39(1): 1-7.

14. Benotsch E, Snipes D, Martin A, Bull S. Sexting, substance use, and sexual risk behavior in young adults. J Adolesc Health. 2013; 52(3):307-13.

15. Folcha C, Casabona J, Sanclemente C, Esteve A, Gonzalez V, HIVTS-TS. Tendencias de la prevalencia del VIH y de las conductas de riesgo asociadas en mujeres trabajadoras del sexo en Cataluña. Gaceta Sanitaria. 2014; 28(3): 196-202.

16. Dessunti E, Advincula A. Factores psicosociales y comportamentales asociados al riesgo de ETS/SIDA entre estudiantes del área de la salud. Rev Latino-am Enfermagem. 2007; 15(2): 267-74.

17. Piña J, Robles S, Rivera B. Instrumento para la evaluación de variables psicológicas y comportamientos sexuales de riesgo en jóvenes de dos centros universitarios de México. Rev Panam Salud Publica/Pan Am J Public Health 2007; 22(5):295-303. 
18. Encuesta Nacional de Demografía y Salud 2010. Febrero 2011. Ministerio de Protección Social. República de Colombia. [citado el 20 de abril de 2016] Disponible en: https://dhsprogram.com/pubs/pdf/FR246/FR246.pdf.

19. Davila M, Piña J. Caracterización, predictores de comportamientos sexuales de riesgo y uso de preservativo en mujeres universitarias. Enseñanza e Investigación en Psicología. 2008; 13(2): 279-99.

20. Declaración de Helsinki de la Asociación Médica. Mundial Principios éticos para las investigaciones médicas en seres humanos [citado el 20 de abril de 2016]. Disponible en: http://www.wma.net/ es/30publications/10policies/b3/17c_es.pdf.

21. República de Colombia Ministerio de Salud. Resolución $\mathrm{N}^{\circ} 008430$ DE 1993 (4 DE OCTUBRE DE 1993) [citado el 20 de abril de 2016]. Disponible en: https:// www.unisabana.edu.co/fileadmin/Documentos/ Investigacion/comite_de_etica/Res_8430_1993_-Salud.pdf.

22. Cortés A, García R, Lazo Z, Bolaños J, Ocho R, Pérez D. Intervención en el conocimiento, creencias y percepción de riesgo sobre infecciones de transmisión sexual y el VIH/SIDA. Medicentro Electrónica. 2014; 18(1):11-8.

23. Staras S, Tobler A, Maldonado M, Cook R. Riskier Sexual Partners Contribute to the Increased Rate of Sexually Transmitted Diseases Among Youth With Substance Use Disorders. Sexually Transmitted Diseases. 2011; 38(5): 413-18.

24. Campo A, Martínez L, Ceballos G. Concordancia entre dos formas de investigar la orientación sexual en adolescentes de Santa Marta, Colombia. Univ. Psychol. 2009; 8(2): 423-28.

25. Arias L, Vásquez M, Dueñas E, García L, Tejada E. Comportamiento sexual y erotismo en estudiantes universitarios, Cali, Colombia. Colombia Médica. 2011; 42(3): 309-18.
26. Gewirtzman A, Bobrick L, Conner K, Tyring S. Epidemiology of Sexually Transmitted Infections. In: Gross G, Tyring S. Sexually Transmitted Infections and Sexually Transmitted Diseases. Springer; 2011.

27. Lomba L, Mendes F. Consumo de drogas, alcohol y conductas sexuales en los ambientes recreativos nocturnos de Portugal. Adicciones. 2009; 21(4):309-26.

28. Ruiz A, Espada J. Consumo de sustancias y conductas sexuales de riesgo para la transmisión del VIH en una muestra de estudiantes universitarios. Anales de Psicología. 2009; 25(2): 344-50.

29. Girón SL, Palacio H, Mateus JC. HIV sexual risk behaviors in youth 15-24 years of age in Cali, Colombia: Do differences exist among neighborhoods?. Colomb Med. 2013; 44(2): 72-9.

30. Escalante L, Cerrón C, Salazar A, Mezones E. Descripción de la Conducta Sexual en adultos jóvenes Limeños. Revista Horizonte Médico. 2008; 8(1): 73-80.

31. Hernández Z, Cruz A. Conductas sexuales riesgosas y adictivas en estudiantes universitarios. Psicología y Salud. 2008; 18(2): 227-36.

32. Ramiro L, Gaspar M, Alves J. Knowledge Attitude and Behaviour Related to Sexually Transmitted Infections in Portuguese School (Adolescent) and College Students. Sexually Transmitted Infections. In: Malla N. In Tech; 2012. pp: 199 - 218. Disponible en: http://cdn. intechopen.com/pdfs/33074.pdf.

33. Estrategia mundial de prevención y control de las Infecciones de Transmisión Sexual 2006-2015. Ginebra. Organización Mundial de la Salud; 2007.

34. Suárez-Colorado Y, Guzmán-García K, Medina-Alfonso L, Ceballos-Ospino G. Características de inteligencia emocional y género en estudiantes de Psicología y Administración de Empresas de una universidad pública de Santa Marta, Colombia: un estudio piloto. Duazary. 2012; 9(2):132-9.

Para citar este artículo: Villafañe-Ferrer L, González-Navarro R. Comportamientos de riesgo y nivel de conocimiento de infecciones de transmisión sexual en estudiantes universitarios. Duazary. 2017 julio; 14 (2): 160 - 168. Doi: http://dx.doi.org/10.21676/2389783X.1964 